$7-24-2020$

\title{
The Comparison Between Maximum Weighted and Trimmed Likelihood Estimator of The Simple Circular Regression Model
}

\author{
Ehab A. Mahmood \\ University of Babylon, Iraq, ehab.mahmood@uobabylon.edu.iq \\ Habshah Midi \\ University Putra Malaysia, Serdang, Malaysia \\ Abdul Ghapor Hussin \\ National Defense University of Malaysia, Kuala Lumpur, Malaysia
}

Follow this and additional works at: https://digitalcommons.wayne.edu/jmasm

Part of the Applied Statistics Commons, Social and Behavioral Sciences Commons, and the Statistical Theory Commons

\section{Recommended Citation}

Mahmood, E. A., Midi, H., \& Hussin, A. G. (2019). The comparison between maximum weighted and trimmed likelihood estimator of the simple circular regression model. Journal of Modern Applied Statistical Methods, 18(2), eP2689. doi: 10.22237/jmasm/1604188920

This Emerging Scholar is brought to you for free and open access by the Open Access Journals at DigitalCommons@WayneState. It has been accepted for inclusion in Journal of Modern Applied Statistical Methods by an authorized editor of DigitalCommons@WayneState. 


\section{EMERGING SCHOLARS}

\section{The Comparison Between Maximum Weighted and Trimmed Likelihood Estimator of the Simple Circular Regression Model}

\author{
Ehab A. Mahmood \\ University of Babylon \\ Babylon, Iraq
}

\author{
Habshah Midi \\ University Putra Malaysia \\ Serdang, Malaysia
}

\author{
Abdul Ghapor Hussin \\ National Defense University of Malaysia \\ Kuala Lumpur, Malaysia
}

The Maximum Likelihood Estimator (MLE) was used to estimate unknown parameters of the simple circular regression model. However, it is very sensitive to outliers in data set. A robust method to estimate model parameters is proposed.

Keywords: Circular regression, maximum likelihood estimator, robust estimation, outliers

\section{Introduction}

The simple circular regression model is proposed to represent the relationship between response and explanatory variables when both of them are circular. The maximum likelihood estimator is considered to estimate the model parameters (Hussin et al., 2004). The Maximum likelihood estimation does not provide reliable estimates for unknown parameters model when data sets have outliers, which is an observation that is markedly different from the majority of a data set (Barnett \& Lewis, 1994).

In the simple circular regression model, there may not be an extreme value due to the circular geometry theory, but not much work was done to overcome the existence of outliers in the simple circular regression model. Abuzaid et al. (2011) suggested using the COVRATIO statistic. Abuzaid et al. (2013) proposed the mean circular error statistic $D M C E c$ to identify outliers in the response variable of a simple circular regression model. Rana et al. (2016) proposed a statistic to identify

doi: 10.22237/jmasm/1604188920 | Accepted: November 16, 2017; Published: July 24, 2020.

Correspondence: Ehab A. Mahmood, ehab.mahmood@uobabylon.edu.iq 


\section{EHAB A. MAHMOOD}

outliers in both response and explanatory variables of the simple circular regression model. Mahmood et al. (2017) introduced RCDy statistic to detect outliers in response variable of the simple circular regression model. However, no robust estimation approach has been proposed to estimate the parameters of the simple circular regression model to handle the presence of outliers in a data set. The robust estimation methods are more resistant to the presence of outliers than classical methods (Hadi \& Luceno, 1997). The purpose of this study is to extend the maximum weighted likelihood estimator and maximum trimmed likelihood estimator to estimate the parameters of the simple circular regression model when the response variable has some outliers.

\section{Simple Circular Regression Model}

When both the response variable $y$ and the explanatory variable $x$ are circular variables, Hussin et al. (2004) decided their linear relationship in a model given by

$$
y_{i}=\alpha+\beta x_{i}+\varepsilon_{i}(\bmod 2 \pi)
$$

where $\alpha$ and $\beta$ are the parameters of the model and $\varepsilon$ is the circular random error, which follows the von Mises distribution with a circular mean 0 and concentration parameter $k$. The von Mises distribution is well known distribution that represents the circular data. It is called the normal distribution for the circular data (Mardia \& Jupp, 2000). The probability density function of von Mises distribution (Fisher, 1993) is

$$
\mathrm{f}(\vartheta, \mu, k)=\frac{1}{2 \pi \mathrm{I}_{0}(k)} \mathrm{e}^{k \cos (\vartheta-\mu)},
$$

where $\vartheta$ is a circular observation, $\mu$ is a mean direction, $k$ is a concentration parameter and $\mathrm{I}_{0}$ denotes the modified Bessel function of the first kind and order zero, which can be defined as

$$
\mathrm{I}_{0}(k)=\frac{1}{2 \pi} \int_{0}^{2 \pi} \mathrm{e}^{k \cos (\vartheta)} d \vartheta
$$

The maximum likelihood estimates of the model parameters (Hussin et al., 2013) are 


\section{MLE AND THE SIMPLE CIRCULAR REGRESSION MODEL}

$$
\hat{\alpha}= \begin{cases}\tan ^{-1}(s / c) & \text { if } s>0, c>0 \\ \tan ^{-1}(s / c)+\pi & \text { if } c<0 \\ \tan ^{-1}(s / c)+2 \pi & \text { if } s<0, c>0\end{cases}
$$

where

$$
\begin{gathered}
s=\sum \sin \left(y_{i}-\hat{\beta} x_{i}\right), \quad c=\sum \cos \left(y_{i}-\hat{\beta} x_{i}\right), \\
\hat{\beta}_{1} \approx \hat{\beta}_{0}+\frac{\sum x_{i} \sin \left(y_{i}-\hat{\alpha}-\hat{\beta}_{0} x_{i}\right)}{\sum x_{i}^{2} \cos \left(y_{i}-\hat{\alpha}-\hat{\beta}_{0} x_{i}\right)} \\
\hat{y}_{i}=\hat{\alpha}+\hat{\beta} x_{i}(\bmod 2 \pi)
\end{gathered}
$$

\section{Robust Estimation}

Robust method is a method less affected by outliers because their effect is reduced by giving small weight to outliers. For example, the observations that are far from the majority of a data set are given either small weights or weights equal to zero (hard rejection of outliers). Hadi and Luceno (1997). Robust estimation methods are proposed here for circular data by down weighting suspected outliers.

\section{Maximum Weighted Likelihood Estimator (MWLE)}

Vander and Neykov (1998) introduced MWLE as one of a robust method to estimate the unknown parameters in the linear regression model. In this section, we extend maximum weighted likelihood estimator to estimate the parameters of the simple circular regression model. The probability density function of von Mises distribution of circular error of the simple circular regression model is defined as follows (Hussin et al., 2004):

$$
\mathrm{f}(\alpha, \beta, \kappa, x, y)=\frac{1}{2 \pi \mathrm{I}_{0}(k)} \mathrm{e}^{k \cos \left(y_{i}-\alpha-\beta x_{i}\right)},
$$

where $0 \leq x, y<2 \pi$, 


\section{EHAB A. MAHMOOD}

$$
\operatorname{lnf}\left(\alpha, \beta, \kappa, x_{i}, y_{i}\right)=\ln \frac{1}{2 \pi \mathrm{I}_{0}(k)}+k \cos \left(y_{i}-\alpha-\beta x_{i}\right) .
$$

The weighted log-likelihood function can be defined as follows:

$$
\begin{aligned}
\ell_{w}(\alpha, \beta, \kappa, x, y) & =\sum_{i=1}^{n} w_{i} \ln \mathrm{f}\left(\alpha, \beta, \kappa, x_{i}, y_{i}\right) \\
& =\sum_{i=1}^{n} w_{i} \ln \frac{1}{2 \pi \mathrm{I}_{0}(k)}+\sum_{i=1}^{n} w_{i} k \cos \left(y_{i}-\alpha-\beta x_{i}\right)
\end{aligned}
$$

The first partial derivative for $\alpha$ is

$$
\begin{gathered}
\frac{\partial}{\partial \alpha} \ell_{w}(\alpha, \beta, \kappa, x, y)=\kappa \sum_{i=1}^{n} w_{i} \sin \left(y_{i}-\alpha-\beta x_{i}\right), \\
\sum_{i=1}^{n} w_{i} \sin \left[\left(y_{i}-\hat{\beta} x_{i}\right)-\hat{\alpha}\right]=0, \\
\sum_{i=1}^{n} w_{i} \sin \left(y_{i}-\hat{\beta} x_{i}\right) \cos (\hat{\alpha})-\sum_{i=1}^{n} w_{i} \cos \left(y_{i}-\hat{\beta} x_{i}\right) \sin (\hat{\alpha})=0, \\
\tan (\hat{\alpha})=\frac{\sum_{i=1}^{n} w_{i} \sin \left(y_{i}-\hat{\beta} x_{i}\right)}{\sum_{i=1}^{n} w_{i} \cos \left(y_{i}-\hat{\beta} x_{i}\right)},
\end{gathered}
$$

where, $w_{i}$ is the weight function.

$$
\begin{gathered}
\hat{\alpha}= \begin{cases}\tan ^{-1}\left(S_{w} / C_{w}\right) & \text { if } S_{w} \geq 0, C_{w}>0 \\
\tan ^{-1}\left(S_{w} / C_{w}\right)+\pi & \text { if } C_{w}<0 \\
\tan ^{-1}\left(S_{w} / C_{w}\right)+2 \pi & \text { if } S_{w}<0, C_{w} \geq 0\end{cases} \\
S_{w}=\sum_{i=1}^{n} w_{i} \sin \left(y_{i}-\hat{\beta} x_{i}\right), \quad C_{w}=\sum_{i=1}^{n} w_{i} \cos \left(y_{i}-\hat{\beta} x_{i}\right) .
\end{gathered}
$$

The first partial derivative for $\beta$ is determined as follows: 


\section{MLE AND THE SIMPLE CIRCULAR REGRESSION MODEL}

$$
\begin{gathered}
\frac{\partial}{\partial \beta} \ell_{w}(\alpha, \beta, \kappa, x, y)=\kappa \sum_{i=1}^{n} w_{i} x_{i} \sin \left(y_{i}-\alpha-\beta x_{i}\right), \\
\sum_{i=1}^{n} w_{i} x_{i} \sin \left(y_{i}-\hat{\alpha}-\hat{\beta} x_{i}\right)=0
\end{gathered}
$$

To estimate $\hat{\beta}$, it can be computed iteratively (Hussin et al., 2004) as

$$
\begin{aligned}
y_{i}-\hat{\alpha}-\hat{\beta} x_{i} & =\left(y_{i}-\hat{\alpha}-\hat{\beta}_{0} x_{i}\right)+x_{i}\left(\hat{\beta}_{0}-\hat{\beta}\right) \\
& =\left(y_{i}-\hat{\alpha}-\hat{\beta}_{0} x_{i}\right)+\Delta x_{i}
\end{aligned}
$$

where $\hat{\beta}_{0}$ is an initial estimate and $\Delta=\hat{\beta}_{0}-\hat{\beta}$.

Thus,

$$
\begin{aligned}
& \sum_{i=1}^{n} w_{i} x_{i} \sin \left(y_{i}-\hat{\alpha}-\hat{\beta} x_{i}\right)=\sum_{i=1}^{n} w_{i} x_{i} \sin \left[\left(y_{i}-\hat{\alpha}-\hat{\beta}_{0} x_{i}\right)+\Delta x_{i}\right]=0 \\
& =\sum_{i=1}^{n} w_{i} x_{i}\left[\sin \left(y_{i}-\hat{\alpha}-\hat{\beta}_{0} x_{i}\right) \cos \left(\Delta x_{i}\right)+\cos \left(y_{i}-\hat{\alpha}-\hat{\beta}_{0} x_{i}\right) \sin \left(\Delta x_{i}\right)\right]=0
\end{aligned}
$$

For small $\Delta, \cos \left(\Delta x_{i}\right) \approx 1$ and $\sin \left(\Delta x_{i}\right) \approx \Delta x_{i}$.

$$
\begin{gathered}
\sum_{i=1}^{n} w_{i} x_{i}\left[\sin \left(y_{i}-\hat{\alpha}-\hat{\beta}_{0} x_{i}\right)+x_{i}\left(\hat{\beta}_{0}-\hat{\beta}\right) \cos \left(y_{i}-\hat{\alpha}-\hat{\beta}_{0} x_{i}\right)\right]=0 \\
\sum_{i=1}^{n} w_{i} x_{i} \sin \left(y_{i}-\hat{\alpha}-\hat{\beta}_{0} x_{i}\right)=\left(\hat{\beta}-\hat{\beta}_{0}\right) \sum_{i=1}^{n} w_{i} x_{i}^{2} \cos \left(y_{i}-\hat{\alpha}-\hat{\beta}_{0} x_{i}\right) \\
\hat{\beta}_{1} \approx \hat{\beta}_{0}+\frac{\sum_{i=1}^{n} w_{i} x_{i} \sin \left(y_{i}-\hat{\alpha}-\hat{\beta}_{0} x_{i}\right)}{\sum_{i=1}^{n} w_{i} x_{i}^{2} \cos \left(y_{i}-\hat{\alpha}-\hat{\beta}_{0} x_{i}\right)}
\end{gathered}
$$

The iteration is continued until the convergence criterion satisfied.

The $w_{i}$ are formulated to give smaller weight to outliers. Hence, the $w_{i}$ are established by firstly developed a robust diagnostic method that can identify outliers in circular data. Mahmood et al. (2017) proposed the $R C D_{y}$ statistic to 


\section{EHAB A. MAHMOOD}

identify outliers in the response variable of the simple circular regression model. They considered the circular distance between circular residuals $c r_{i}$ and its median as a statistic to detect outliers. The propose statistic is denoted as

$$
\left[R C D_{i}\right]_{y}=\pi-|\pi-| c r_{i}-\operatorname{med}(c r) \mid
$$

and the cut-off point is denoted as cut $R C D_{y}=\max \left[R C D_{i}\right]_{y}$.

Consider this statistic and its cut-off point to compute weights as

$$
w_{i}= \begin{cases}1 & \text { if }\left[R C D_{i}\right]_{y} \leq \operatorname{cut} R C D_{y} \\ \operatorname{cut} R C D_{y} /\left[R C D_{i}\right]_{y} & \text { if }\left[R C D_{i}\right]_{y}>\operatorname{cut} R C D_{y}\end{cases}
$$

\section{Maximum Trimmed Likelihood Estimator (MTLE)}

Hadi and Luceno (1997) proposed MTLE as a way to make the MLE robust, and here it is extended to estimate parameters of the simple circular regression model. Following their idea, consider $R C D_{y}$ statistic as a measure for trimming (hard rejection of outliers) as

$$
w_{i}= \begin{cases}1 & \text { if }\left[R C D_{i}\right]_{y} \leq \mathrm{cut} R C D_{y} \\ 0 & \text { if }\left[R C D_{i}\right]_{y}>\operatorname{cut} R C D_{y}\end{cases}
$$

\section{Simulation}

Simulation studies were undertaken to examine the performance of our robust approaches to estimate model parameters of the simple circular regression model. A set of explanatory variables were generated as $[x \sim \operatorname{vM}(\pi / 4,10)]$, the circular random error $[\varepsilon \sim \operatorname{vM}(0, k)]$, and $\alpha=0, \beta=1$ for each sample size $n$ and concentration parameter $k$. Consider three sample sizes, $n=20$, 60, and 100, with six values of concentration parameter, $k=2,3,5,6,8$, and 10, and two ratios of contamination in the response variable, $10 \%$ and $20 \%$. The response variable is contaminated according to

$$
y_{c(i)}=y_{(i)}+\lambda \pi \bmod (2 \pi)
$$




\section{MLE AND THE SIMPLE CIRCULAR REGRESSION MODEL}

where $y_{c(i)}$ is the contaminated circular observation and $\lambda$ is the degree of contamination such that $(0 \leq \lambda \leq 1)$. Here, we consider $(\lambda=0.8)$. The MLE, MWLE, and MTLE are then applied to the data. Replicate these combination 10,000 times. The performance of the three methods are evaluated based on the bias, variance, and mean square error (MSE) of the estimates. They are computed (Khokan et al., 2013; Fan et al., 1998) as bias $=\hat{\gamma}-\gamma$, where $\gamma$ is a model parameter,

$$
\operatorname{var}(\hat{\beta})=\frac{\sum_{j=1}^{R}\left(\hat{\beta}_{j}-\text { mean }(\hat{\beta})\right)^{2}}{(R-1)},
$$

and $R$ is the number of replications.

In the circular data, it is known that the difference between two circular data points should computed the formula given by Mardia and Jupp (2000). The formula to compute variance of $(\hat{\alpha})$ is

$$
\operatorname{var}(\hat{\alpha})=\frac{\sum_{j=1}^{R}\left(\pi-|\pi-| \hat{\alpha}_{j}-\operatorname{mean}(\hat{\alpha}) \mid\right)^{2}}{(R-1)}
$$

$M S E=$ bias $^{2}+$ var.

The results for clean, $10 \%$, and $20 \%$ of contaminated data of bias, variance, and MSE of $\hat{\alpha}$ and $\hat{\beta}$ for sample size 60 are shown in Figures 1-3, respectively. As to be expected, there is not much difference among the MLE, MWLE, and MTLE for clean data. However, the bias of $\hat{\alpha}$ and $\hat{\beta}$ of MLE are more affected by outliers and have large values of bias for high ratios of contamination. The bias of $\hat{\alpha}$ and $\hat{\beta}$ of MWLE are less than the bias of MLE with concentration parameter greater than three. The MTLE gives the best results of bias with $10 \%$ and $20 \%$ of contamination except in the case of small values of concentration parameter. This is because for smaller concentration parameter, the circular data will be more spread around the circumference, which make it difficult to identify outliers Collett (1980). 


\section{EHAB A. MAHMOOD}
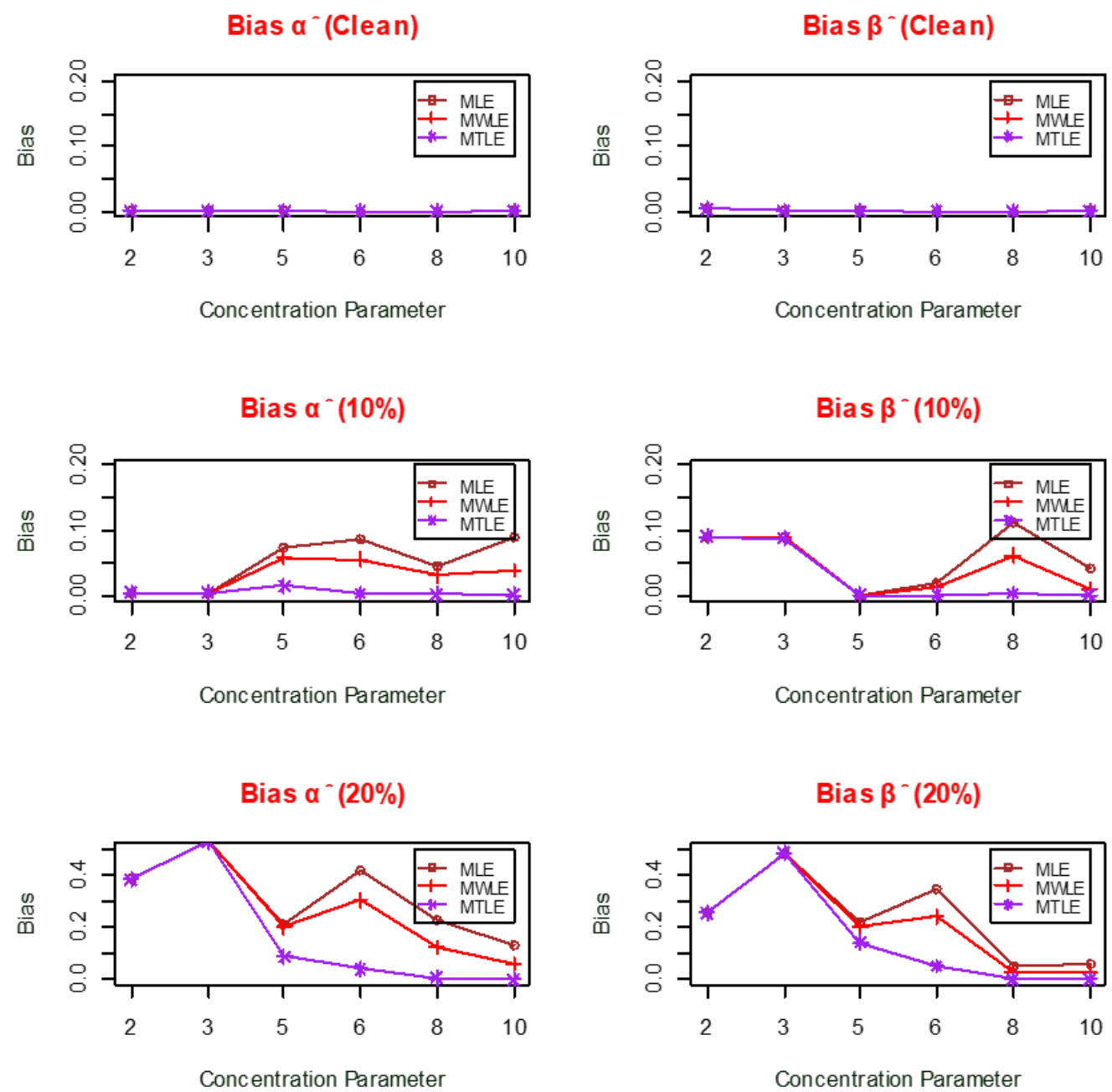

Figure 1. Bias ( $\hat{\alpha}$ and $\hat{\beta})$ of clean and contaminated data with $10 \%$ and $20 \%(n=60$, $\alpha=0, \beta=1)$

As noted in Figure 2, the variance of $\hat{\alpha}$ and $\hat{\beta}$ of MTLE is slightly better than the variance of MLE with $10 \%$ of contamination. However, the performance of MTLE is better than that of MLE with $20 \%$ of contamination for concentration parameter greater than three. 


\section{MLE AND THE SIMPLE CIRCULAR REGRESSION MODEL}
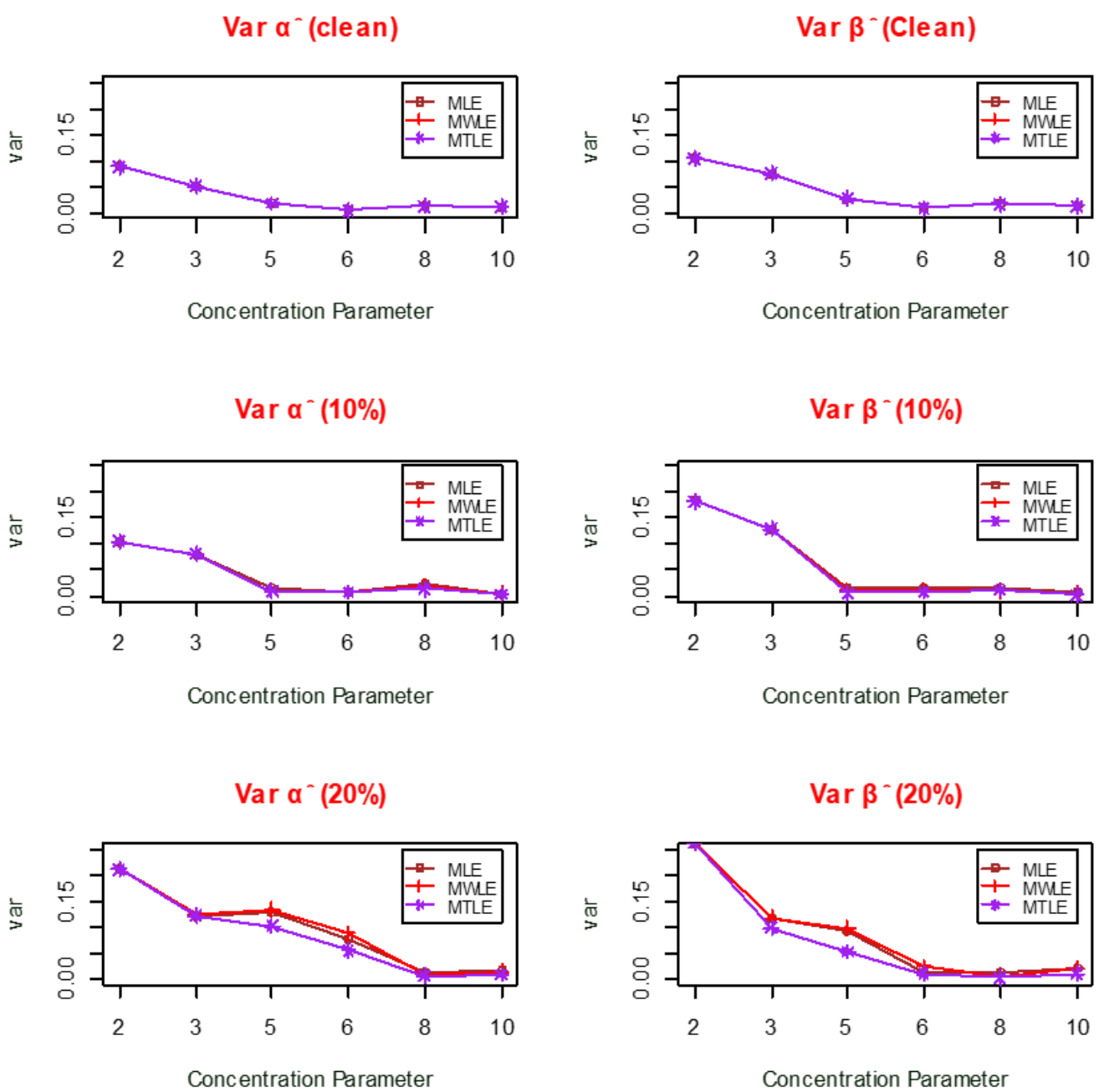

Figure 2. Variance ( $\hat{\alpha}$ and $\hat{\beta}$ ) of clean and contaminated data with $10 \%$ and $20 \%$ $(n=60, \alpha=0, \beta=1)$

As noted in Figure 3, the MSE of MTLE is slightly less than the MSE of the MLE with $10 \%$. However, both MWLE and MTLE are very successful to down weight outliers for concentration parameter greater than three with $20 \%$ of contamination. Above all, the MTLE gives the best results followed by the MWLE and MLE. Due to space limitation, only results for $n=60$ are presented, because results for $n=20$ and 100 were consistent. 


\section{EHAB A. MAHMOOD}
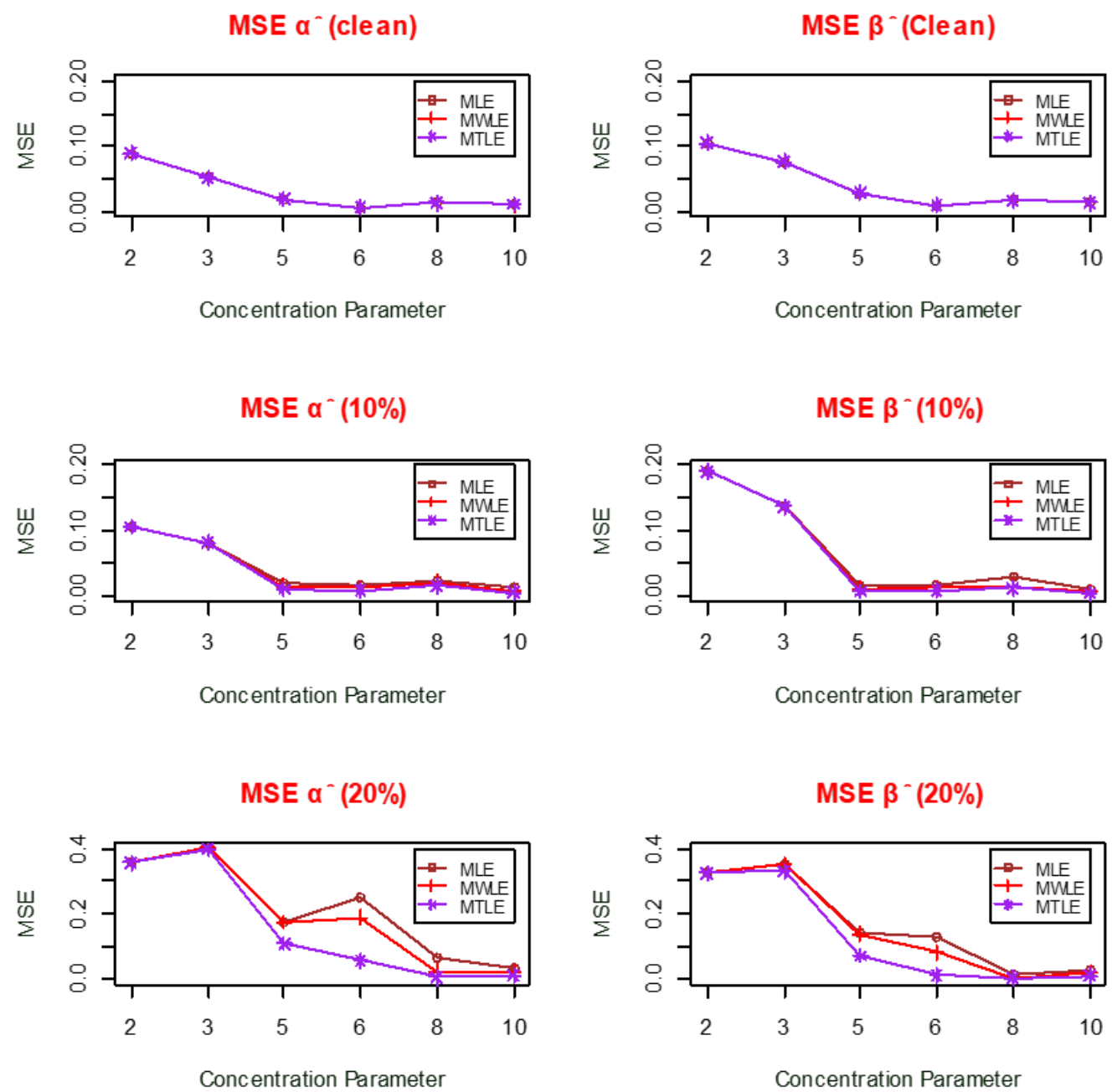

Figure 3. MSE ( $\hat{\alpha}$ and $\hat{\beta}$ ) of clean and contaminated data with $10 \%$ and $20 \%(n=60$, $\alpha=0, \beta=1)$

\section{Example}

Consider the wind directions data which is taken from Hussin et al. (2004). The data set has response and explanatory circular variables of a sample size $(n=129)$. The variables represent the measurements by radians were recorded along the Holderness coastline (the Humberside coast of North Sea, United Kingdom) by using HF radar system (explanatory variable) and anchored wave buoy (response 


\section{MLE AND THE SIMPLE CIRCULAR REGRESSION MODEL}

Table 1. Estimates and variance of MLE, weighted and trimmed

\begin{tabular}{|c|c|c|c|c|c|c|}
\hline \multirow[b]{2}{*}{ Parameter } & \multicolumn{2}{|c|}{ MLE } & \multicolumn{2}{|c|}{ MWLE } & \multicolumn{2}{|c|}{ MTLE } \\
\hline & Est. & Var. & Est. & Var. & Est. & Var. \\
\hline$\hat{\alpha}$ & 4.951 & 0.2207 & 4.936 & 0.1990 & 5.179 & 0.2882 \\
\hline$\hat{\beta}$ & 1.430 & 0.6274 & 1.422 & 0.0585 & 1.366 & 0.0442 \\
\hline
\end{tabular}

variable). The observations 38 and 111 are found to be outliers. In order to investigate the performance of MLE and the proposed robust methods (MWLE and MTLE), a bootstrapping technique was used as an alternative approach to compute the parameter estimates and the variance of the parameters estimate of the model. Because the explanatory variable is assumed to be fixed, the fixed $\mathrm{X}$ bootstrapping was based on 1,000 replications. The results are shown in Table 1. The variance of $\hat{\alpha}$ of MTLE is slightly larger than MLE and MWLE. However, this is not a concern, because the parameter estimates of $\hat{\alpha}$ does not give important meaning. Nonetheless, both MWLE and MTLE are better than MLE because their variance of $\hat{\beta}$ are smaller than MLE. The variance of $\hat{\beta}$ is more important than variance of $\hat{\alpha}$ as a measure for comparison because $\hat{\beta}$ relates with explanatory variable. Hence, the MTLE is the best robust approach because it has the smallest variance of $\hat{\beta}$.

\section{Conclusion}

Two robust methods, MWLE and MTLE, were proposed to estimate the parameters of the simple circular regression model, which is important because the traditional maximum likelihood estimator (MLE) is sensitive to outliers. The proposed robust methods are very successful to estimate model parameters at high ratio of contamination and concentration parameter greater than three. According to the simulation and example results, the MTLE gives the best result to estimate model parameters of the simple circular regression model when the response variable has some outliers.

\section{References}

Abuzaid, A. H., Hussin, A. G., \& Mohamed, I. B. (2013). Detection of outliers in simple circular regression models using the mean circular error 


\section{EHAB A. MAHMOOD}

statistic. Journal of Statistical Computation and Simulation, 83(2), 269-277. doi: 10.1080/00949655.2011.602679

Abuzaid, A. H., Mohamed, I., Hussin, A.G., \& Rambli, A. (2011). COVARATIO statistic for simple circular regression model. Chiang Mai Journal of Science, 38(3), 321-330. Retrieved from https://epg.science.cmu.ac.th/ejournal/journalDetail.php?journal_id=542

Barnett, V., \& Lewis, T. (1994). Outliers in statistical data ( $3^{\text {rd }}$ edition). New York: Wiley.

Collett, D. (1980). Outliers in circular data. Journal of the Royal Statistical Society, Series C: Applied Statistics, 29(1), 50-57. doi: 10.2307/2346410

Fan, J., Farmen, M., \& Gijbels, I. (1998). Local maximum likelihood estimation and inference. Journal of the Royal Statistical Society, Series B: Statistical Methodology, 60(3), 591-608. doi: 10.1111/1467-9868.00142

Fisher, N. I. (1993). Statistical analysis of circular data. Cambridge: Cambridge University Press.

Hadi, A. S., \& Luceno, A. (1997). Maximum trimmed likelihood estimators: A unified approach, examples and algorithms. Computational Statistics \& Data Analysis, 25(3), 251-272. doi: 10.1016/S0167-9473(97)00011-X

Hussin, A. G., Abuzaid, A. H., Ibrahim, A. I. N., \& Rambli, A. (2013). Detection of outliers in the complex linear regression model. Sains Malaysiana, 42(6), 869-874. Retrieved from http://www.ukm.my/jsm/pdf_files/SM-PDF-42-62013/21\%20A.G.\%20Hussin.pdf

Hussin, A. G., Fieller, N. R. J., \& Stillman, E. C. (2004). Linear regression for circular variables with application to directional data. Journal of Applied Science and Technology, 9(1), 1-6. doi: 10.4314/jast.v9i1.17435

Khokan, M. R., Bari, W., \& Khan, J. A. (2013). Weighted maximum likelihood approach for robust estimation: Weibull model. Dhaka University Journal of Science, 61(2), 153-156. doi: 10.3329/dujs.v61i2.17061

Mahmood, E. A., Midi, H., Rana, S., \& Hussin, A. G. (2017). Robust circular distance and its application in the identification of outliers in the simple circular regression model. Asian Journal of Applied Sciences. 10(3), 126-133. doi: 10.3923/ajaps.2017.126.133

Mardia, K. V., \& Jupp, P. (2000). Directional statistics. London: Wiley. doi: 10.1002/9780470316979

Rana, S., Mahmood, E. A., Midi, H., \& Hussin, A. G. (2016). Robust detection of outliers in both response and explanatory variables of the simple 


\section{MLE AND THE SIMPLE CIRCULAR REGRESSION MODEL}

circular regression model. Malaysian Journal of Mathematical Sciences, 10(3), 399-414. Retrieved from

https://einspem.upm.edu.my/jurnal/index.php/mjms/article/view/379

Vander, D. L., \& Neykov, N. M. (1998). About regression estimators with high breakdown point. Statistics, 32(2), 111-129. doi:

10.1080/02331889808802657 